\title{
EFFICIENT ALGORITHMS FOR PERIODIC HERMITE SPLINE INTERPOLATION
}

\author{
G. PLONKA AND M. TASCHE
}

\begin{abstract}
Periodic Hermite spline interpolants on an equidistant lattice are represented by the Bézier technique as well as by the $B$-spline method. Circulant matrices are used to derive new explicit formulas for the periodic Hermite splines of degree $m$ and defect $r(1 \leq r \leq m)$. Applying the known de Casteljau algorithm and the de Boor algorithm, respectively, we obtain new efficient real algorithms for periodic Hermite spline interpolation.
\end{abstract}

\section{INTRODUCTION}

This paper deals with periodic Hermite spline interpolation on the equidistant lattice $\mathbb{Z}$. Other approaches to this problem use Euler-Frobenius polynomials and complex line integrals (see [4-6]) or Euler-Frobenius polynomials and circulant matrices (see $[7,8]$ ). Similar to $[7,8]$, we prefer a real-algebraic method for periodic Hermite spline interpolation. Contrary to [4-8], we apply a vectorial Bézier technique and later a periodic $B$-spline method in this note. This leads to new efficient real algorithms for periodic Hermite spline interpolation. These methods are based on the de Casteljau algorithm and the de Boor algorithm, respectively. Both procedures possess a low arithmetic complexity. Further, one can see that the generalized Euler-Frobenius polynomials are very important for periodic Hermite spline interpolation. Note that our methods can be extended to periodic Hermite spline interpolation with shifted nodes too.

\section{Preliminaries}

In this paper we use standard notations. First we recall some facts concerning circulant matrices, which form the background of the considerations in $\S \S 3$ and 4 (cf. [2]).

Let $N \in \mathbb{N}(N>1)$ be fixed. For $\mathbf{a}=\left(a_{1}, \ldots, a_{N}\right)^{T} \in \mathbb{R}^{N}$, let

$$
\operatorname{circa}^{T}:=\left(a_{j-i+1}\right)_{i, j=1}^{N}=\left[\begin{array}{cccc}
a_{1} & a_{2} & \cdots & a_{N} \\
a_{N} & a_{1} & \cdots & a_{N-1} \\
\vdots & \vdots & & \vdots \\
a_{2} & a_{3} & \cdots & a_{1}
\end{array}\right]
$$

Received November 9, 1990.

1991 Mathematics Subject Classification. Primary 65D07, 41A15, 15 A09.

$K e y$ words and phrases. Periodic Hermite spline interpolation, Bézier technique, $B$-spline technique, Bernstein polynomials, circulant matrices, Euler-Frobenius polynomials. 
denote the associated circulant $(N, N)$-matrix. Note that the subscripts must be calculated modulo $N$ here and in the following. For arbitrary $\mathbf{a}=$ $\left(a_{1}, \ldots, a_{N}\right)^{T}, \mathbf{b}=\left(b_{1}, \ldots, b_{N}\right)^{T} \in \mathbb{R}^{N}$, and $\alpha \in \mathbb{R}$, we have

$$
\begin{gathered}
\operatorname{circ} \mathbf{a}^{T}+\operatorname{circ} \mathbf{b}^{T}=\operatorname{circ}(\mathbf{a}+\mathbf{b})^{T}, \\
\alpha \operatorname{circ} \mathbf{a}^{T}=\operatorname{circ}(\alpha \mathbf{a})^{T}, \quad\left(\operatorname{circ} \mathbf{a}^{T}\right)\left(\operatorname{circ} \mathbf{b}^{T}\right)=\operatorname{circ} \mathbf{c}^{T}
\end{gathered}
$$

with $\mathbf{c}=\left(c_{1}, \ldots, c_{N}\right)^{T}$ and

$$
c_{i}:=\sum_{j=1}^{N} a_{j} b_{i-j+1} \quad(i=1, \ldots, N) .
$$

Let $\mathbf{e}_{i}:=\left(\delta_{1 i}, \ldots, \delta_{N i}\right)^{T}(i=1, \ldots, N)$. Let $\mathbf{V}:=\operatorname{circ} \mathbf{e}_{2}^{T}$ denote the fundamental circulant matrix. Then we have

$$
\mathbf{V}^{k}=\operatorname{circ} \mathbf{e}_{k+1}^{T} \quad(k=1, \ldots, N-1), \quad \mathbf{V}^{N}=\mathbf{I}, \quad \mathbf{V}^{T}=\mathbf{V}^{-1}=\mathbf{V}^{N-1} .
$$

Note that $\mathbf{V}$ is a cyclic shift matrix, i.e., $\mathbf{V a}=\left(a_{2}, \ldots, a_{N}, a_{1}\right)^{T}$. It is clear that

$$
\operatorname{circ} \mathbf{a}^{T}=a_{1} \mathbf{I}+a_{2} \mathbf{V}+\cdots+a_{N} \mathbf{V}^{N-1} .
$$

Introducing $p(\lambda):=a_{1}+a_{2} \lambda+\cdots+a_{N} \lambda^{N-1}$, we see that $\operatorname{circ} \mathbf{a}^{T}=p(\mathbf{V})$. Hence all circulant $(N, N)$-matrices commute [2, p. 68].

Now we simplify a recent result of [7] and present a shorter proof.

Theorem 1. Let $1 \leq k \leq N-1$, and let $\lambda_{j} \in \mathbb{C}$ with $\lambda_{j}^{N} \neq 1 \quad(j=1, \ldots, k)$ be given. Furthermore, let

$$
p(\lambda):=\prod_{j=1}^{k}\left(\lambda-\lambda_{j}\right)
$$

Then $p(\mathbf{V})$ is nonsingular, and its inverse is given by

$$
p(\mathbf{V})^{-1}=\operatorname{circ}\left(b_{1}, \ldots, b_{N}\right),
$$

where the $b_{n}(n=1, \ldots, N)$ are the divided differences

$$
b_{n}:=\left[\tau^{N-n}\left(1-\tau^{N}\right)^{-1} ; \lambda_{1}, \ldots, \lambda_{k}\right] .
$$

Proof. 1. First we show the existence of a polynomial

$$
q(\lambda)=b_{1}+b_{2} \lambda+\cdots+b_{N} \lambda^{N-1}
$$

with the property $p(\lambda) q(\lambda) \equiv 1 \bmod \left(\lambda^{N}-1\right)$.

Set

$$
\begin{aligned}
Q(\lambda, \tau) & :=\left(\lambda^{N-1}+\lambda^{N-2} \tau+\cdots+\tau^{N-1}\right)\left(1-\tau^{N}\right)^{-1} \\
& =(\lambda-\tau)^{-1}+\left(\lambda^{N}-1\right)(\lambda-\tau)^{-1}\left(1-\tau^{N}\right)^{-1} .
\end{aligned}
$$

Using divided differences with respect to the variable $\tau$, we get by induction on $k$ that

$$
\begin{gathered}
{\left[(\lambda-\tau)^{-1} ; \lambda_{1}, \ldots, \lambda_{k}\right]=p(\lambda)^{-1},} \\
{\left[(\lambda-\tau)^{-1}\left(1-\tau^{N}\right)^{-1} ; \lambda_{1}, \ldots, \lambda_{k}\right]=r(\lambda) p(\lambda)^{-1},}
\end{gathered}
$$

where $r(\lambda)$ is a polynomial of degree $\leq k-1$. Then

$$
q(\lambda):=\left[Q(\lambda, \tau) ; \lambda_{1}, \ldots, \lambda_{k}\right]
$$


possesses the coefficients (1.1) by (1.2). Hence, it follows from (1.2) that

$$
p(\lambda) q(\lambda)=1+\left(\lambda^{N}-1\right) r(\lambda) \text {. }
$$

2. By (1.3) we obtain immediately that $p(\mathbf{V}) q(\mathbf{V})=\mathbf{I}$, i.e., $p(\mathbf{V})^{-1}=$ $q(\mathbf{V})$.

Remark. For odd $N>1, k=1$ and $\lambda_{1}=-1$, it follows from Theorem 1 that $\mathbf{V}+\mathbf{I}$ is nonsingular, and its inverse is

$$
(\mathbf{V}+\mathbf{I})^{-1}=\frac{1}{2} \operatorname{circ}(1,-1,1,-1, \ldots, 1) \text {. }
$$

\section{HERMITE SPLINE INTERPOLATION PROBLEM}

Let $N, m, r \in \mathbb{N}$ with $N, m>1$ and $r \leq m$ be fixed. By $S_{m, N}^{m-r}$ we denote the linear space of all $N$-periodic real functions $s \in C^{m-r}(\mathbb{R})$ with

$$
s(j-1+t)=p_{j}(t), \quad p_{j}=p_{j+N} \in \mathbb{P}_{m}
$$

for all $t \in[0,1]$ and for all $j \in \mathbb{Z}$, where $\mathbb{P}_{m}$ denotes the set of all real polynomials of degree $\leq m$ defined on $[0,1]$. The elements of $S_{m, N}^{m-r}$ are called $N$-periodic spline functions of degree $m$ and defect $r$ on the equidistant lattice $\mathbb{Z}$. Note that $\operatorname{dim} S_{m, N}^{m-r}=r N$.

We consider the following $N$-periodic Hermite spline interpolation problem: For given data $y_{j}^{(k)} \in \mathbb{R}(j \in \mathbb{Z} ; k=0, \ldots, r-1)$ with $y_{j}^{(k)}=y_{j+N}^{(k)}$, we wish to find an $N$-periodic spline function $s \in S_{m, N}^{m-r}$ satisfying the Hermite interpolation conditions

$$
\begin{aligned}
s^{(k)}(j) & =y_{j}^{(k)} & (j \in \mathbb{Z} ; k & =0, \ldots, l), \\
s^{(k)}(j-0) & =y_{j}^{(k)} & (j \in \mathbb{Z} ; k & =l+1, \ldots, r-1),
\end{aligned}
$$

with $l:=\min (r-1, m-r)$.

Remark. In the case $2 r \leq m+1$, we obtain the classical Hermite spline interpolation problem (2.1). If $2 r \geq m+1$, then (2.1) corresponds to separated 2-point Hermite interpolation problems, since $m-r+1$ (and $r$, respectively) Hermite data on the left (and right, respectively) end point of every subinterval $[j, j+1]$ are prescribed (cf. [8]).

\section{Solution By the BÉZIER techNiQue}

By an idea of [4], the periodic splines can be represented in a transparent form as polynomial vectors such that one can work with cyclic shifts and circulant matrices. Obviously, $s \in S_{m, N}^{m-r}$ can be characterized by the polynomial vector $\mathbf{p}:=\left(p_{N}, \ldots, p_{1}\right)^{T} \in \mathbb{P}_{m}^{N}$ with the spline conditions (cf. [4-8])

$$
\mathbf{p}^{(k)}(0)=\mathbf{V p}^{(k)}(1) \quad(k=0, \ldots, m-r) .
$$

With the notation $\mathbf{y}^{(k)}:=\left(y_{N}^{(k)}, \ldots, y_{1}^{(k)}\right)^{T} \in \mathbb{R}^{N} \quad(k=0, \ldots, r-1)$, the Hermite interpolation problem $(2.1)$ is equivalent to computing $\mathbf{p} \in \mathbb{P}_{m}^{N}$ such that the spline conditions (3.1) and the Hermite interpolation conditions

$$
\mathbf{p}^{(k)}(1)=\mathbf{y}^{(k)} \quad(k=0, \ldots, r-1)
$$

are fulfilled (cf. [4-8]). 
Every $\mathbf{p} \in \mathbb{P}_{m}^{N}$ can be expressed in the form

$$
\mathbf{p}=\sum_{i=0}^{m} \mathbf{a}_{i} B_{i}^{m}
$$

with the Bernstein polynomials

$$
B_{i}^{m}(t):=\left(\begin{array}{c}
m \\
i
\end{array}\right)(1-t)^{m-i} t^{i} \quad(i=0, \ldots, m ; t \in[0,1])
$$

and with vectors $\mathbf{a}_{i} \in \mathbb{R}^{N}$. The representation (3.3) is called a Bézier polynomial of degree $m$. The vectors $\mathbf{a}_{i}$ are the Bézier coefficients of $\mathbf{p}$ (cf. [1]). From (3.3) it follows that the $k$ th derivative of $\mathbf{p}$ is

$$
\mathbf{p}^{(k)}=\frac{m !}{(m-k) !} \sum_{i=0}^{m-k}\left(\Delta^{k} \mathbf{a}_{i}\right) B_{i}^{m-k} \quad(k=1, \ldots, m)
$$

with the $k$ th differences

$$
\Delta^{k} \mathbf{a}_{i}:=\sum_{j=0}^{k}(-1)^{k-j}\left(\begin{array}{c}
k \\
j
\end{array}\right) \mathbf{a}_{i+j} \quad(i=0, \ldots, m-k) .
$$

As an immediate consequence of (3.4) we obtain that

$$
\mathbf{p}^{(k)}(0)=\frac{m !}{(m-k) !} \Delta^{k} \mathbf{a}_{0}, \quad \mathbf{p}^{(k)}(1)=\frac{m !}{(m-k) !} \Delta^{k} \mathbf{a}_{m-k} .
$$

By (3.5) it follows from the spline conditions (3.1) and from the Hermite interpolation conditions (3.2) that

$$
\begin{aligned}
\Delta^{k} \mathbf{a}_{0} & =\mathbf{V} \Delta^{k} \mathbf{a}_{m-k} & & (k=0, \ldots, m-r), \\
\Delta^{k} \mathbf{a}_{m-k} & =\frac{(m-k) !}{m !} \mathbf{y}^{(k)} & & (k=0, \ldots, r-1) .
\end{aligned}
$$

The linear equations (3.6) are equivalent to the following system:

$$
\begin{gathered}
\sum_{j=0}^{k}(-1)^{k-j}\left(\begin{array}{l}
k \\
j
\end{array}\right) \mathbf{a}_{j}=\frac{(m-k) !}{m !} \mathbf{V} \mathbf{y}^{(k)} \quad(k=0, \ldots, l), \\
\sum_{j=0}^{k}(-1)^{k-j}\left(\begin{array}{l}
k \\
j
\end{array}\right) \mathbf{a}_{m-j}=\frac{(m-k) !}{m !}(-1)^{k} \mathbf{y}^{(k)} \quad(k=0, \ldots, r-1),
\end{gathered}
$$

and, if $2 r \leq m$,

$$
\sum_{j=0}^{k}(-1)^{k-j}\left(\begin{array}{l}
k \\
j
\end{array}\right)\left(\mathbf{a}_{j}-\mathbf{V} \mathbf{a}_{m-k+j}\right)=\mathbf{0} \quad(k=r, \ldots, m-r) .
$$

Since the inverse of the lower triangular matrix $\left((-1)^{k-j}\left(\begin{array}{l}k \\ j\end{array}\right)\right)_{k, j=0}^{l}$ is equal to the lower triangular matrix $\left(\left(\begin{array}{l}j \\ k\end{array}\right)\right)_{j, k=0}^{l}$, the system (3.7) possesses the solution

$$
\mathbf{a}_{j}=\mathbf{V} \sum_{k=0}^{j} \frac{(m-k) !}{m !}\left(\begin{array}{l}
j \\
k
\end{array}\right) \mathbf{y}^{(k)} \quad(j=0, \ldots, l) .
$$


Analogously, the system (3.8) has the solution

$$
\mathbf{a}_{m-j}=\sum_{k=0}^{j}(-1)^{k} \frac{(m-k) !}{m !}\left(\begin{array}{l}
j \\
k
\end{array}\right) \mathbf{y}^{(k)} \quad(j=0, \ldots, r-1) .
$$

In the case $2 r \geq m+1$, the system (3.9) does not occur and all vectors $\mathbf{a}_{0}, \ldots, \mathbf{a}_{m-r}, \mathbf{a}_{m-r+1}, \ldots, \mathbf{a}_{m}$ are uniquely determined by (3.10) and (3.11). Thus, the periodic Hermite spline interpolation problem under consideration is uniquely solvable. If $2 r \leq m$, then the system (3.9) can be reduced to a system of $m+1-2 r$ linear equations in $m+1-2 r$ unknown vectors $\mathbf{a}_{r}, \ldots, \mathbf{a}_{m-r}$ by substituting the vectors (3.10) and (3.11) into (3.9). The solvability of this system will be discussed in the following. For practical reasons we consider in detail only the cases $m \leq 5$.

Theorem 2. Let $N, m, r \in \mathbb{N}$ with $N, m>1$ and $r \leq m \leq 5$ be given. The $N$-periodic Hermite spline interpolation problem on the equidistant lattice $\mathbb{Z}$ possesses a unique solution $\mathbf{p} \in \mathbb{P}_{m}^{N}$ of the form (3.3) in the cases $(m, r)=$ $(2,2),(3,1),(3,2),(3,3),(4,3),(4,4),(5,1),(5,2),(5,3),(5,4)$, $(5,5)$ or $(m, r)=(2,1),(4,1)$ with odd $N$. If $2 r \geq m+1$, then all Bézier coefficients of $\mathbf{p}$ are given by (3.10) and (3.11). If $2 r \leq m$, then $\mathbf{a}_{0}=V_{\mathbf{y}} \mathbf{y}^{(0)}$ and $\mathbf{a}_{m}=\mathbf{y}^{(0)}$; furthermore, we have in the case:

(i) $(m, r)=(2,1)$ with odd $N$ :

$$
\mathbf{a}_{1}=2 \mathbf{W}^{-1} \mathbf{V} \mathbf{y}^{(0)}
$$

with $\mathbf{W}:=\mathbf{V}+\mathbf{I}$,

(ii) $(m, r)=(3,1)$ :

$$
\mathbf{a}_{1}=2 \mathbf{W}^{-1} \mathbf{V}(2 \mathbf{V}+\mathbf{I}) \mathbf{y}^{(0)}, \quad \mathbf{a}_{2}=2 \mathbf{W}^{-1} \mathbf{V}(\mathbf{V}+2 \mathbf{I}) \mathbf{y}^{(0)}
$$

with $\mathbf{W}:=\mathbf{V}^{2}+4 \mathbf{V}+\mathbf{I}$,

(iii) $(m, r)=(4,1)$ with odd $N$ :

$$
\begin{aligned}
& \mathbf{a}_{1}=2 \mathbf{W}^{-1} \mathbf{V}\left(4 \mathbf{V}^{2}+7 \mathbf{V}+\mathbf{I}\right) \mathbf{y}^{(0)}, \\
& \mathbf{a}_{2}=4 \mathbf{W}^{-1} \mathbf{V}\left(\mathbf{V}^{2}+4 \mathbf{V}+\mathbf{I}\right) \mathbf{y}^{(0)}, \\
& \mathbf{a}_{3}=2 \mathbf{W}^{-1} \mathbf{V}\left(\mathbf{V}^{2}+7 \mathbf{V}+4 \mathbf{I}\right) \mathbf{y}^{(0)}
\end{aligned}
$$

with $\mathbf{W}:=\left(\mathbf{V}^{2}+10 \mathbf{V}+\mathbf{I}\right)(\mathbf{V}+\mathbf{I})$,

(iv) $(m, r)=(5,1)$ :

$$
\begin{aligned}
& \mathbf{a}_{1}=2 \mathbf{W}^{-1} \mathbf{V}\left(8 \mathbf{V}^{3}+33 \mathbf{V}^{2}+18 \mathbf{V}+\mathbf{I}\right) \mathbf{y}^{(0)}, \\
& \mathbf{a}_{2}=4 \mathbf{W}^{-1} \mathbf{V}\left(2 \mathbf{V}^{3}+15 \mathbf{V}^{2}+12 \mathbf{V}+\mathbf{I}\right) \mathbf{y}^{(0)}, \\
& \mathbf{a}_{3}=4 \mathbf{W}^{-1} \mathbf{V}\left(\mathbf{V}^{3}+12 \mathbf{V}^{2}+15 \mathbf{V}+2 \mathbf{I}\right) \mathbf{y}^{(0)}, \\
& \mathbf{a}_{4}=2 \mathbf{W}^{-1} \mathbf{V}\left(\mathbf{V}^{3}+18 \mathbf{V}^{2}+33 \mathbf{V}+8 \mathbf{I}\right) \mathbf{y}^{(0)}
\end{aligned}
$$

with $\mathbf{W}:=\mathbf{V}^{4}+26 \mathbf{V}^{3}+66 \mathbf{V}^{2}+26 \mathbf{V}+\mathbf{I}$,

(v) $(m, r)=(5,2)$ :

$$
\begin{aligned}
& \mathbf{a}_{1}=\mathbf{V y}^{(0)}+\frac{1}{5} \mathbf{V} \mathbf{y}^{(1)}, \quad \mathbf{a}_{2}=\mathbf{W}^{-1}\left(-4 \mathbf{V}^{2} \mathbf{y}^{(0)}+\frac{4}{5} \mathbf{V}(\mathbf{I}-3 \mathbf{V}) \mathbf{y}^{(1)}\right), \\
& \mathbf{a}_{3}=\mathbf{W}^{-1}\left(-4 \mathbf{V} \mathbf{y}^{(0)}-\frac{4}{5} \mathbf{V}(\mathbf{V}-3 \mathbf{I}) \mathbf{y}^{(1)}\right), \quad \mathbf{a}_{4}=\mathbf{y}^{(0)}-\frac{1}{5} \mathbf{y}^{(1)}
\end{aligned}
$$

with $\mathbf{W}:=\mathbf{V}^{2}-6 \mathbf{V}+\mathbf{I}$. 
Proof. In the cases $2 r \leq m \leq 5$, the solvability of the reduced system (3.9) with known vectors $\mathbf{a}_{0}, \ldots, \mathbf{a}_{m-r}, \mathbf{a}_{m-r+1}, \ldots, \mathbf{a}_{m}$ must be investigated. For the sake of simplicity, we discuss here only the case $(m, r)=(5,2)$. From (3.10) and (3.11) it follows that

$$
\begin{gathered}
\mathbf{a}_{0}=\mathbf{V} \mathbf{y}^{(0)}, \quad \mathbf{a}_{1}=\mathbf{V} \mathbf{y}^{(0)}+\frac{1}{5} \mathbf{V} \mathbf{y}^{(1)}, \\
\mathbf{a}_{4}=\mathbf{y}^{(0)}-\frac{1}{5} \mathbf{y}^{(1)}, \quad \mathbf{a}_{5}=\mathbf{y}^{(0)} .
\end{gathered}
$$

Substituting these results into (3.9), we get the reduced system

$$
\begin{gathered}
\mathbf{a}_{2}-\mathbf{V} \mathbf{a}_{3}=\frac{4}{5} \mathbf{V} \mathbf{y}^{(1)}, \\
(\mathbf{V}-\mathbf{3 I}) \mathbf{a}_{2}+(\mathbf{I}-\mathbf{3} \mathbf{V}) \mathbf{a}_{3}=-4 \mathbf{V} \mathbf{y}^{(0)}
\end{gathered}
$$

This yields

$$
\begin{aligned}
& \mathbf{W} \mathbf{a}_{2}=-4 \mathbf{V}^{2} \mathbf{y}^{(0)}+\frac{4}{5} \mathbf{V}(\mathbf{I}-3 \mathbf{V}) \mathbf{y}^{(1)} \\
& \mathbf{W} \mathbf{a}_{3}=-4 \mathbf{V} \mathbf{y}^{(0)}-\frac{4}{5} \mathbf{V}(\mathbf{V}-3 \mathbf{I}) \mathbf{y}^{(1)}
\end{aligned}
$$

with the circulant $(N, N)$-matrix $\mathbf{W}:=\mathbf{V}^{2}-6 \mathbf{V}+\mathbf{I}$. Since $p(\lambda):=\lambda^{2}-6 \lambda+1$ does not vanish at one of the $N$ th roots of unity, $\mathbf{W}=p(\mathbf{V})$ is nonsingular (see [2, p. 89] or Theorem 1) and $\mathbf{W}^{-1}$ can be computed with the help of Theorem 1 .

The de Casteljau algorithm (cf. [1]) evaluates the vector $\mathbf{p}(t) \in \mathbb{R}^{N} \quad(t \in$ $[0,1])$ of the form (3.3) step by step for $r=1, \ldots, m$ by convex combinations

$$
\begin{aligned}
& \mathbf{a}_{i}^{(r)}(t):=(1-t) \mathbf{a}_{i}^{(r-1)}(t)+t \mathbf{a}_{i+1}^{(r-1)}(t) \quad(i=0, \ldots, m-r), \\
& \mathbf{a}_{i}^{(0)}(t):=\mathbf{a}_{i} \quad(i=0, \ldots, m) .
\end{aligned}
$$

After $m$ steps, (3.12) generates the single vector $\mathbf{a}_{0}^{(m)}(t)=\mathbf{p}(t) \in \mathbb{R}^{N}$. The de Casteljau algorithm (3.12) works in parallel, is numerically stable [3], and requires $m(m+1) N$ real multiplications and $m(m+1) N / 2$ real additions.

Together with Theorems 1 and 2, we obtain an efficient algorithm for the computation of $N$-periodic Hermite spline interpolants. If $2 r \geq m+1$, then our algorithm requires only $O(N)$ arithmetic operations. If $2 r \leq m$, and if $N$ is a power of 2 , then we can compute the products of the circulant $(N, N)$ matrix $\mathbf{W}^{-1}$ and some $N$-dimensional vectors (see Theorem 2) with the help of the fast Fourier transform, so that our algorithm involves only $O\left(N \log _{2} N\right)$ arithmetic operations.

\section{Solution By THE $B$-SPLINE TECHNIQUe}

In the following we solve the Hermite spline interpolation problem (2.1) by periodic $B$-splines.

Let $N, m, r \in \mathbb{N}$ with $N, m>1$ and $r \leq m$ be fixed. By $\mathbf{y}^{(k)}:=$ $\left(y_{N}^{(k)}, \ldots, y_{1}^{(k)}\right)^{T} \in \mathbb{R}^{N} \quad(k=0, \ldots, r-1)$ we denote again the given Hermite data. Consider equidistant knots with multiplicity $r$ :

$$
x_{j+r k}:=k \quad(k \in \mathbb{Z} ; j=0, \ldots, r-1) .
$$


Assume that $x_{r+m} \leq N$. Let $B_{k}^{m, r} \in C^{m-r}(\mathbb{R})$ denote the normalized $B$-spline of degree $m$ and defect $r$ with the knots $x_{k}, x_{k+1}, \ldots, x_{k+m+1}$. Then the $N$-periodic $B$-spline $P_{k}^{m, r}$ is given by

$$
P_{k}^{m, r}(x):=\sum_{n=-\infty}^{\infty} B_{k+n N}^{m, r}(x)
$$

Note that the $N$-periodic $B$-splines

$$
P_{j+r k}^{m, r}(x)=P_{j}^{m, r}(x-k) \quad(j=0, \ldots, r-1 ; k=0, \ldots, N-1)
$$

form a basis of the spline space $S_{m, N}^{m-r}$.

First we consider the case $r=1$ of Lagrange spline interpolation for odd $m$ $(1<m \leq N-1)$. If $s \in S_{m, N}^{m-1}$ of the form

$$
s(x)=\sum_{k=0}^{N-1} c_{k+1} P_{0}^{m, 1}(x-k)
$$

fulfills the Lagrange interpolation condition (2.1) with $r=1$, then one has

$$
\sum_{i=0}^{m-1} B_{0}^{m, 1}(i+1) \mathbf{V}^{i} \mathbf{c}=\mathbf{y}^{(0)}
$$

with $\mathbf{c}:=\left(c_{N}, \ldots, c_{1}\right)^{T} \in \mathbb{R}^{N}$. In terms of the Euler-Frobenius polynomial

$$
H_{m, 1}(\lambda):=m ! \sum_{i=0}^{m-1} B_{0}^{m, 1}(i+1) \lambda^{i},
$$

it follows that

$$
H_{m, 1}(\mathbf{V}) \mathbf{c}=m ! \mathbf{y}^{(0)}
$$

As we know [10], $H_{m, 1}$ possesses only simple negative zeros. Since $H_{m, 1}(-1) \neq$ 0 for odd $m, H_{m, 1}(\mathbf{V})$ is nonsingular. Thus, we obtain the solution by

$$
\mathbf{c}=m ! H_{m, 1}(\mathbf{V})^{-1} \mathbf{y}^{(0)} \text {. }
$$

Comparing with Theorem 2, we see that (4.2) has a similar structure as the corresponding result in $\S 3$.

Remark. By definition, $H_{m, 1}$ is a monic polynomial of degree $m-1$, since we have

For example, we get

$$
B_{0}^{m, 1}(1)=B_{0}^{m, 1}(m)=(m !)^{-1} .
$$

$$
\begin{aligned}
& H_{2,1}(\lambda)=\lambda+1 \\
& H_{3,1}(\lambda)=\lambda^{2}+4 \lambda+1 \\
& H_{4,1}(\lambda)=\lambda^{3}+11 \lambda^{2}+11 \lambda+1, \\
& H_{5,1}(\lambda)=\lambda^{4}+26 \lambda^{3}+66 \lambda^{2}+26 \lambda+1 .
\end{aligned}
$$

For the sake of simplicity, we consider now only the case $r=2$ of Hermite spline interpolation for odd $m=2 n+1 \quad(1 \leq n \leq N-1)$. If $s \in S_{m, N}^{m-2}$ of the form

$$
s(x)=\sum_{k=0}^{N-1}\left(c_{k+1} P_{0}^{m, 2}(x-k)+d_{k+1} P_{1}^{m, 2}(x-k)\right)
$$


fulfils the Hermite interpolation condition (2.1) with $r=2$, then we get

$$
\begin{aligned}
& \sum_{i=0}^{n-1}\left(B_{0}^{m, 2}(i+1) \mathbf{V}^{i} \mathbf{c}+B_{1}^{m, 2}(i+1) V^{i} \mathbf{d}\right)=\mathbf{y}^{(0)} \\
& \sum_{i=0}^{n-1}\left(\left(B_{0}^{m, 2}\right)^{\prime}(i+1) \mathbf{V}^{i} \mathbf{c}+\left(B_{1}^{m, 2}\right)^{\prime}(i+1) \mathbf{V}^{i} \mathbf{d}\right)=\mathbf{y}^{(1)}
\end{aligned}
$$

with $\mathbf{c}:=\left(c_{N}, \ldots, c_{1}\right)^{T} \in \mathbb{R}^{N}$ and $\mathbf{d}:=\left(d_{N}, \ldots, d_{1}\right)^{T} \in \mathbb{R}^{N}$. Defining the generalized Euler-Frobenius polynomial by

$$
H_{m, 2}(\lambda):=\frac{(n+1)(n !)^{4}}{m}\left|\begin{array}{ll}
\sum_{i=0}^{n-1} B_{0}^{m, 2}(1+i) \lambda^{i} & \sum_{\substack{i=0 \\
n-1}}^{n-1} B_{1}^{m, 2}(1+i) \lambda^{i} \\
\sum_{i=0}^{n-1}\left(B_{0}^{m, 2}\right)^{\prime}(1+i) \lambda^{i} & \sum_{i=0}^{n-1}\left(B_{1}^{m, 2}\right)^{\prime}(1+i) \lambda^{i}
\end{array}\right|,
$$

we conclude immediately that

$$
\begin{aligned}
& H_{m, 2}(\mathbf{V}) \mathbf{c}=\frac{(n+1)(n !)^{4}}{m} \sum_{i=0}^{n-1}\left(\left(B_{1}^{m, 2}\right)^{\prime}(1+i) \mathbf{V}^{i} \mathbf{y}^{(0)}-B_{1}^{m, 2}(1+i) \mathbf{V}^{i} \mathbf{y}^{(1)}\right), \\
& H_{m, 2}(\mathbf{V}) \mathbf{d}=\frac{(n+1)(n !)^{4}}{m} \sum_{i=0}^{n-1}\left(B_{0}^{m, 2}(1+i) \mathbf{V}^{i} \mathbf{y}^{(1)}-\left(B_{0}^{m, 2}\right)^{\prime}(1+i) \mathbf{V}^{i} \mathbf{y}^{(0)}\right)
\end{aligned}
$$

Hence, our periodic Hermite spline interpolation problem is uniquely solvable if and only if the circulant matrix $H_{m, 2}(\mathbf{V})$ is nonsingular. For $m=3,5,7,9$, $H_{m, 2}(\mathbf{V})$ is nonsingular, since all zeros of $H_{m, 2}$ are positive and $\neq 1$ (see [6]).

Remark. By definition, $H_{m, 2}$ is a monic polynomial of degree $m-3$, since we have

$$
\begin{aligned}
\left|\begin{array}{cc}
B_{0}^{m, 2}(1) & B_{1}^{m, 2}(1) \\
\left(B_{0}^{m, 2}\right)^{\prime}(1) & \left(B_{1}^{m, 2}\right)^{\prime}(1)
\end{array}\right| & =\left|\begin{array}{cc}
B_{0}^{m, 2}(n-1) & B_{1}^{m, 2}(n-1) \\
\left(B_{0}^{m, 2}\right)^{\prime}(n-1) & \left(B_{1}^{m, 2}\right)^{\prime}(n-1)
\end{array}\right| \\
& =\frac{m}{(n+1)(n !)^{4}} .
\end{aligned}
$$

For example, we get

$$
\begin{aligned}
& H_{3,2}(\lambda)=1 \\
& H_{5,2}(\lambda)=1-6 \lambda+\lambda^{2}, \\
& H_{7,2}(\lambda)=1-72 \lambda+262 \lambda^{2}-72 \lambda^{3}+\lambda^{4}, \\
& H_{9,2}(\lambda)=1-522 \lambda+13839 \lambda^{2}-38732 \lambda^{3}+13839 \lambda^{4}-522 \lambda^{5}+\lambda^{6} .
\end{aligned}
$$

The zeros of these polynomials are tabulated in [6].

Using divided differences and symmetry properties, we can precompute the values $B_{k}^{m, 2}(1+i)$ and $\left(B_{k}^{m, 2}\right)^{\prime}(1+i)(k=0,1 ; i=0, \ldots, n-1)$. The inverse of $H_{m, 2}(\mathbf{V})$ can be computed by Theorem 1. Once c and d are calculated, we obtain the $N$-periodic Hermite spline with the help of the known de Boor algorithm. 
Note that this $B$-spline approach can be used also for $r>2$. Then the generalized Euler-Frobenius polynomial $H_{m, r}$ is introduced by

$$
H_{m, r}(\lambda):=c_{m, r}\left|\begin{array}{ccc}
\sum_{i=0}^{m-1} B_{0}^{m, r}(1+i) \lambda^{i} & \ldots & \sum_{i=0}^{m-1} B_{r-1}^{m, r}(1+i) \lambda^{i} \\
\vdots & & \vdots \\
\sum_{i=0}^{m-1}\left(B_{0}^{m, r}\right)^{(r-1)}(1+i) \lambda^{i} & \cdots & \sum_{i=0}^{m-1}\left(B_{r-1}^{m, r}\right)^{(r-1)}(1+i) \lambda^{i}
\end{array}\right|
$$

with

$$
c_{m, r}^{-1}:=\left|\begin{array}{ccc}
B_{0}^{m, r}(m) & \cdots & B_{r-1}^{m, r}(m) \\
\vdots & & \vdots \\
\left(B_{0}^{m, r}\right)^{(r-1)}(m) & \cdots & \left(B_{r-1}^{m, r}\right)^{(r-1)}(m)
\end{array}\right| .
$$

Comparing the Bézier technique with the $B$-spline method, we observe that the corresponding coefficients have a similar structure. In both cases, the arithmetic complexity is determined mainly by the computation of $H_{m, r}(\mathbf{V})^{-1}$ and by multiplication of the circulant matrix $H_{m, r}(\mathbf{V})^{-1}$ with a certain vector. Note that there exists a linear relationship between the Bézier coefficients and the $B$-spline coefficients. Namely, for $r=1$ we get the Bézier coefficients $a_{k}$ $(k=0, \ldots, m)$ from the vectors c of $B$-spline coefficients (see $\S 4)$ by

$$
\begin{aligned}
& \mathbf{a}_{k}=\frac{1}{m !} \sum_{j=0}^{k}\left(\begin{array}{l}
k \\
j
\end{array}\right) H_{m-j, 1}(\mathbf{V}) \mathbf{V}(\mathbf{I}-\mathbf{V})^{j} \mathbf{c} \quad(k=0, \ldots, m-1), \\
& \mathbf{a}_{m}=\frac{1}{m !} H_{m, 1}(\mathbf{V}) \mathbf{c} .
\end{aligned}
$$

Conversely, we have

$$
\mathbf{c}=m ! H_{m, 1}(\mathbf{V})^{-1} \mathbf{V}^{T} \mathbf{a}_{0}=m ! H_{m, 1}(\mathbf{V})^{-1} \mathbf{a}_{m} .
$$

Finally, we note that our method can be extended to the periodic Hermite spline interpolation with nonequidistant nodes, to nonperiodic Hermite spline interpolation, to Hermite spline interpolation with shifted nodes, and to cardinal Hermite spline interpolation on an equidistant lattice (see [7]).

\section{Generalized Euler-Frobenius polynomials}

Assume that $m=2 n+1 \geq 2 r-1 \geq 1$. In $\S 4$ we defined the generalized Euler-Frobenius polynomials $H_{m, r}$ by means of $B$-splines. In [6-9], the corresponding Euler-Frobenius polynomial is introduced by the following determinant of order $m-r+1$ :

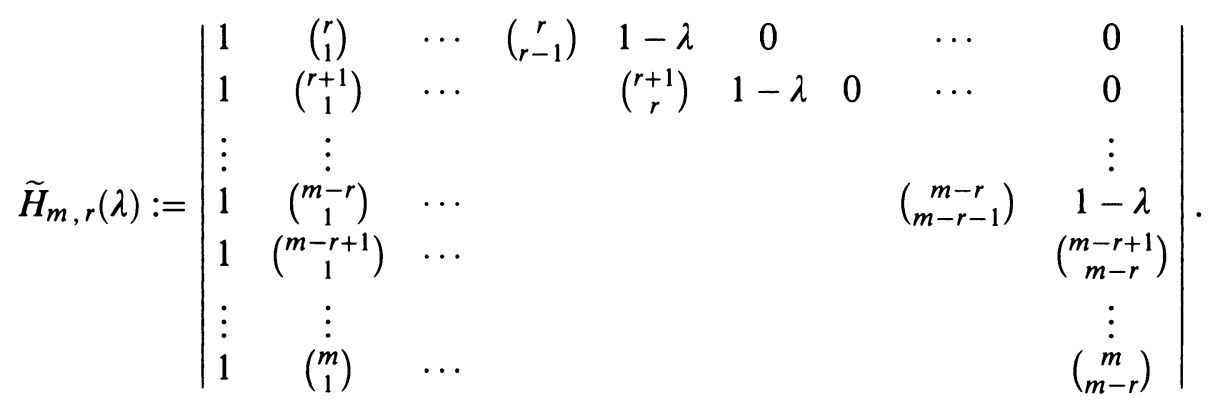


Then $\widetilde{H}_{m, r}$ is a monic polynomial of even degree $m+1-2 r$. Now we prove, with the help of the theory of eigensplines [9], that $H_{m, r}=\widetilde{H}_{m, r}$.

For the sake of simplicity, we discuss in detail only the case $r=2$. Let $S_{m}^{m-2}$ denote the linear space of cardinal splines $s \in C^{m-2}(\mathbb{R})$ with $s \mid[j, j+1] \in \mathbb{P}_{m}$ for all $j \in \mathbb{Z}$. In terms of the $B$-splines $B_{0}^{m, 2}$ and $B_{1}^{m, 2}$ (see $\S 4$ ), every $s \in S_{m}^{m-2}$ can be uniquely represented in the form

$$
s(x)=\sum_{j=-\infty}^{\infty} a_{j} B_{0}^{m, 2}(x-j)+\sum_{j=-\infty}^{\infty} b_{j} B_{1}^{m, 2}(x-j)
$$

with constants $a_{j}, b_{j}(j \in \mathbb{Z})$. Let $N_{m}^{m-2}:=\left\{s \in S_{m}^{m-2} ; s(j)=s^{\prime}(j)=0\right.$ $(j \in \mathbb{Z})\}$. Then we have (see $[9$, p. 46])

$$
\operatorname{dim} N_{m}^{m-2}=m-3 .
$$

Now $s \in N_{m}^{m-2}(s \neq 0)$ is called an eigenspline of $S_{m}^{m-r}[9$, p. 46], if the functional equation $s(x+1)=\mu s(x)$ is fulfilled for some constant $\mu$. We call $\mu$ the corresponding eigenvalue. In [9, pp. 46-47] it is proved that the $m-3$ zeros of the polynomial $\widetilde{H}_{m, 2}$ are precisely the eigenvalues of $S_{m}^{m-2}$. Now we show:

Lemma. The zeros of $H_{m, 2}$ are precisely the eigenvalues of $S_{m}^{m-2}$.

Proof. If $s \in N_{m}^{m-2}$ is an eigenspline with the eigenvalue $\mu \quad(\mu \neq 0)$, then it follows from (5.1) and $s(x+1)=\mu s(x)$ that $a_{j+1}=\mu a_{j}$ and $b_{j+1}=\mu b_{j}$ $(j \in \mathbb{Z})$. Therefore, $a_{j}=a_{0} \mu^{j}$ and $b_{j}=b_{0} \mu^{j} \quad(j \in \mathbb{Z})$, where $\left|a_{0}\right|+\left|b_{0}\right|>0$. Thus we find that

$$
s(x)=a_{0} \sum_{j=-\infty}^{\infty} \mu^{j} B_{0}^{m, 2}(x-j)+b_{0} \sum_{j=-\infty}^{\infty} \mu^{j} B_{1}^{m, 2}(x-j) .
$$

For $x \in[n, n+1]$, we obtain

$$
s(x)=a_{0} \sum_{j=0}^{n} \mu^{j} B_{0}^{m, 2}(x-j)+b_{0} \sum_{j=0}^{n} \mu^{j} B_{1}^{m, 2}(x-j) .
$$

From $s(n)=s^{\prime}(n)=0$, it follows that

$$
\begin{aligned}
& b_{0} \sum_{j=0}^{n-1} \mu^{j} B_{0}^{m, 2}(j+1)+a_{0} \sum_{j=0}^{n-1} \mu^{j} B_{1}^{m, 2}(j+1)=0, \\
& b_{0} \sum_{j=0}^{n-1} \mu^{j}\left(B_{0}^{m, 2}\right)^{\prime}(j+1)+a_{0} \sum_{j=0}^{n-1} \mu^{j}\left(B_{1}^{m, 2}\right)^{\prime}(j+1)=0,
\end{aligned}
$$

since we have

$$
\begin{gathered}
B_{0}^{m, 2}(n+1-x)=B_{1}^{m, 2}(x), \quad B_{1}^{m, 2}(n+1-x)=B_{0}^{m, 2}(x), \\
B_{k}^{m, 2}(n+1)=\left(B_{k}^{m, 2}\right)^{\prime}(n+1)=0 \quad(k=0,1) .
\end{gathered}
$$

From (5.3), we conclude that $H_{m, 2}(\mu)=0$.

Conversely, if $\mu$ is a zero of $H_{m, 2}$ and if $a_{0}, b_{0}$ is a nontrivial solution of (5.3), then the function (5.2) is an eigenspline with the eigenvalue $\mu$. 
Since $\widetilde{H}_{m, 2}$ and $H_{m, 2}$ are monic polynomials of degree $m-3$ with the same zeros, we get $\widetilde{H}_{m, 2}=H_{m, 2}$. Similarly, it can be shown that $\widetilde{H}_{m, r}=H_{m, r}$ for $r \geq 1$.

Remark. Let $m=2 n+1 \geq 2 r-1 \geq 1$. All zeros $\mu_{j}(j=1, \ldots, 2 n-2 r+2)$ of $\widetilde{H}_{m, r}$ are real, simple, and have the sign of $(-1)^{r}$. The zeros can be arranged as follows:

$$
0<\left|\lambda_{1}\right|<\cdots<\left|\lambda_{n-r+1}\right|<1<\left|\lambda_{n-r+2}\right|<\cdots<\left|\lambda_{2 n-2 r+2}\right|,
$$

where $\lambda_{1} \lambda_{2 n-2 r+2}=\cdots=\lambda_{n-r+1} \lambda_{n-r+2}=1$. The value $(-1)^{r}$ is not a zero of $\widetilde{H}_{m, r}$ (see [9, p. 47]).

\section{BIBLIOGRAPHY}

1. W. Böhm, G. Farin, and J. Kahmann, A survey of curve and surface methods in CAGD, Comput. Aided Geom. Design 1 (1984), 1-60.

2. P. J. Davis, Circulant matrices, Wiley, New York, 1979.

3. R. T. Farouki and V. T. Rajan, On the numerical condition of polynomials in Bernstein form, Comput. Aided Geom. Design 4 (1987), 191-216.

4. G. Meinardus and G. Merz, Zur periodischen Spline-Interpolation, Spline-Funktionen (K. Böhmer, G. Meinardus, and W. Schempp, eds.), Bibliographisches Institut, Mannheim, 1974, pp. 177-195.

5. __ Hermite-Interpolation mit periodischen Spline-Funktionen, Numerical Methods of Approximation Theory (L. Collatz, G. Meinardus, and H. Werner, eds.), Birkhäuser, Basel, 1980, pp. 200-210.

6. G. Merz and W. Sippel, Zur Konstruktion periodischer Hermite-Interpolationssplines bei äquidistanter Knotenverteilung, J. Approx. Theory 54 (1988), 92-106.

7. M. Reimer, Cardinal Hermite-spline-interpolation on the equidistant lattice, Numer. Math. 56 (1989), 345-357.

8. __ Zur reellen Darstellung periodischer Hermite-Spline-Interpolierender bei äquidistantem Gitter mit Knotenshift, Splines in Numerical Analysis (J. W. Schmidt and H. Späth, eds.), Akademie-Verlag, Berlin, 1989, pp. 125-134.

9. I. J. Schoenberg, Cardinal spline interpolation, SIAM, Philadelphia, PA, 1973.

10. D. Siepmann, Kardinale Spline-Interpolation bezüglich äquidistant verteilter Knoten, Dissertation, Dortmund, 1984.

FACHBEREICh Mathematik, Universität Rostock, UniVersitätsPlatz 1, 0-2500 Rostock, GERMANY 